\title{
Head-up display with dynamic depth-variable viewing effect
}

Kun Li ${ }^{\mathrm{a}}$, Ye Geng ${ }^{\mathrm{a}}$, Ali Özgür Yöntem ${ }^{\mathrm{a}}$, Daping Chu ${ }^{\mathrm{a},{ }^{*}}$, Valerian Meijering $^{\mathrm{b}}$, Eduardo Dias ${ }^{\mathrm{b}}$, Lee Skrypchuk

${ }^{\text {a }}$ Centre for Photonic Devices and Sensors, University of Cambridge, 9 JJ Thomson Avenue, Cambridge CB3 OFA, United Kingdom

b Jaguar Land Rover, National Automotive Innovation Centre, Lord Bhattacharyya Way, University of Warwick, Coventry, CV4 7AL, United Kingdom

*Corresponding author, email: dpc31@cam.ac.uk

\section{Highlights}

- A head-up display with a local dynamic depth-variable viewing effect for attentions is developed and demonstrated.

- An optical design utilising an electrically tuneable liquid lens is proposed to vary the image depth and magnification of the selected display area.

- The eye-box of the head-up display is enlarged to accommodate driver's head movement, and the brightness of the displayed image is maintained to be uniform across the eye-box.

\begin{abstract}
Head-Up Displays (HUDs) can reduce duration and frequency of drivers looking away from traffic scenes, but information contents of different importance are usually displayed at the same time in contemporary HUD models. Such configurations increase the time that a driver searches for more critical information and it is essential that the said information can quickly attract driver's attention without affecting his focus on the road. We introduce an alternative approach of displaying critical information with a variable depth in a designated local area of a HUD image. The variations are engineered to create a dynamic pop-up effect for hazard warnings, such as a car exceeding the speedlimit or approaching certain road signs. The image depth of the corresponding area is designed to vary by about half a metre and the image size by 1.4 times for a natural viewing experience, using an offthe-shelf liquid lens with electrically tuneable focus depths. The HUD optics can be adjusted to have an extended eye-box to accommodate driver's head movement and a uniformity image brightness across the eye-box.
\end{abstract}

\section{Keywords}


Head-Up Display; Depth-variable; Liquid lens; Eye-box; Dynamic viewing; Warning sign

\section{Introduction}

Every year more than 1.25 million lives are cut short and between 20-50 million more people are injured because of road traffic crashes, according to the World Health Organisation [1]. Most of these crashes are caused by the driver's lack of attention or distractions [2,3], which severely reduces driver's situation awareness of the surrounding environment [4]. About $80 \%$ of driving-safety information are received visually to support safe driving practices [5]. In order to reduce the duration and frequency that drivers look away from the road, Head-Up Displays (HUDs) were developed to present essential driving information in the driver's line of sight $[6,7]$. HUD is an important safety and assistive feature in modern cars $[5,8]$, which distracts drivers the least if compared with the usual navigation devices based on conventional flat panel displays such as Liquid Crystal Displays (LCDs) [9].

Since its early installation in 1988, a HUD has been an add-on option in luxury brands and high-end car models [10]. With the HUD technology widely available for safety and convenience reasons, the market has now extended into the sector of mid-ranged and sporty car models. In recent commercial reports, the global HUD market was valued at 1-2 billion USD in 2016, with an annual growth rate of over $25 \%$ in the next five to ten years $[11,12]$, within which the automotive sector accounts for a significant market share over the forecasted period.

Commercial HUDs can be divided into two main categories, one displays information on a separate and fixed optical screen on top of the dashboard, namely combiner HUDs, and the other displays directly on the front windscreen, namely windscreen HUDs. Combiner HUDs display information at a fixed location with a limited field-of-view, and we will focus only on windscreen HUDs in this study.

Augmented reality (AR) has been an important focus for the recent HUD development $[5,6,13-16]$. It aims to blend the displayed information into the physical world and it can bring more benefits to drivers than a conventional HUD [17-19], such as effective warning signs which are conspicuous to the driver when driving through the clutter in both quiet and busy environments [20]. An AR HUD can also help to improve human performance [21], such as an increased response time through AR cues, according to tests done on simulators [14].

In the meantime, works on conventional HUDs have looked into the variations in the displayed contents and locations for fast response times. It was shown that highlighted display regions in a HUD could be found by the driver faster than non-highlighted ones [22]. Another study demonstrated displaying warning signs in case of speeding could reduce the distraction and reaction time compared with displaying numbers or the combination of numbers and graphics [23]. Furthermore, the displayed 
information was found to be preferential when placed at about $5^{\circ}$ to the left or right of the centre for fast responses [24].

Most HUDs nowadays still have their contents (essential vehicle information and navigation arrows) displayed on a single 2D image plane at a fixed depth, typically 2-2.5 $\mathrm{m}$ away from the driver [25]. Such a configuration is passive and increases the time that a driver searches for more critical information. A smarter system is needed so that the said information can quickly attract driver's attention without affecting his focus on the road. A recent study shows that dynamic variations of the displayed image depth is effective for speed compliance [2], this is similar to that of LED speed signs in road-work zones, which flashes and gets larger and closer as cars approach.

In this work, a HUD with a dynamic-content design is presented, which continuously changes depth of the displayed image for an immediate response from the driver. The optics are designed to provide an intuitive HUD image depth variation and a comfortable viewing angle for the driver. The developed HUD is demonstrated on a car windscreen to deliver speed warning signs with a pop-up effect. The displayed images are captured during the variation process and compared with the static counterpart. Optical performances and limitations of the HUD, such as eye-box size and brightness uniformity, are measured and discussed.

\section{Optical design}

The variation of image depth is achieved by using a liquid lens, Optotune EL-16-40-TC Dietikon Switzerland, its focal length can be tuned by applying an electric current. Similar devices have been adopted on proof-of-concept head-mounted display (HMD) demonstrators to minimise the accommodation-convergence conflict and improve viewer's comfort [26-28], but the setups are too heavy to be employed in wearable applications.

The optical design for image depth variation consists of a liquid lens (L-Lens), an achromatic lens (ALens) and a Fresnel lens (F-Lens), respectively. The L-Lens is based on a shape-changing polymer lens with the focal length controlled by the current flowing through the coil of the actuator [29]. It has an optical power range tuneable between -4.5 and 5.5 dioptres, which corresponds to a plano-concave lens with a focal length from $-222 \mathrm{~mm}$ to $-\infty$ and a plano-convex lens with the focal length from $182 \mathrm{~mm}$ to $+\infty$. The L-Lens has a $5 \mathrm{~ms}$ response time and a $25 \mathrm{~ms}$ settling time, and more than $24 \mathrm{~Hz}$ of video rate can be easily achieved. However, the L-Lens has a very small aperture $(16 \mathrm{~mm})$. To overcome the limitation imposed by the small aperture, the A-Lens is used to generate an enlarged virtual image of the L-Lens. The F-Lens is used to further increase the effective L-Lens aperture, with the additional benefit of its thin form factor, light weight and convenience to cut into a preferred size. 
The three lenses are arranged according to Figure 1 , with distance $\left(d_{1}\right)$ separating the L-Lens and ALens and distance $\left(d_{2}\right)$ separating the A-Lens and F-Lens respectively. Based on the thin lens equation $\frac{1}{s_{0}}+\frac{1}{s_{i}}=\frac{1}{f}$, the image distance $\left(s_{i 1}\right)$ of Image 1 is obtained from the L-Lens focal length $\left(f_{1}\right)$ and the object distance $\left(\mathrm{s}_{01}\right)$, as illustrated in Figure $1(\mathrm{a})$. After passing through all three lenses, a resultant image (Image 3 ) is formed at the image distance $\left(s_{i 3}\right)$ of $46.6 \mathrm{~mm}$ with respect to the F-Lens and a magnification ratio of -2.25 with respect to the object image. The negative sign indicates an inverted image with respect to the original object.

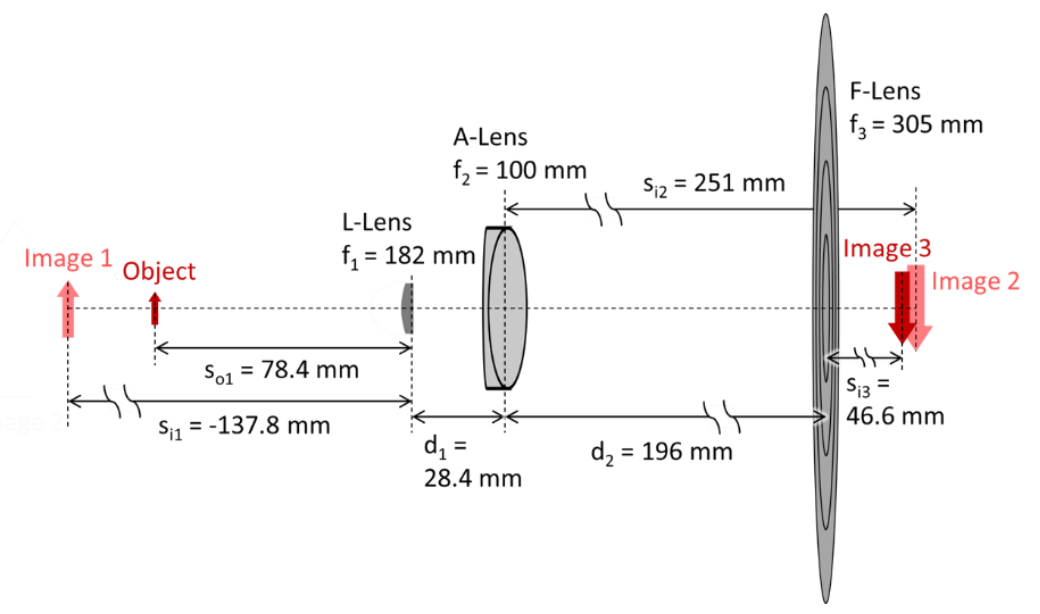

(a)

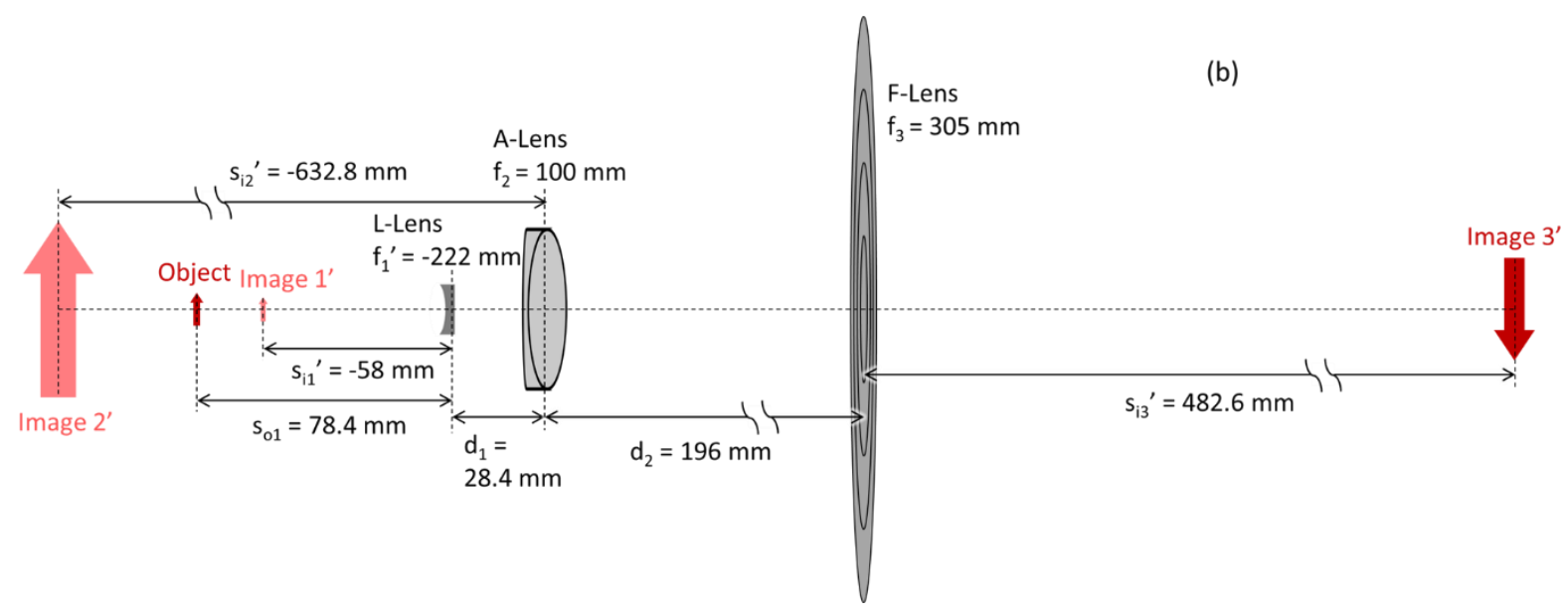

Figure 1. Schematics of the optical design with three lenses, achieving the intended image depth and size variations where the L-Lens focal length is set to (a) $182 \mathrm{~mm}$ and (b) $-222 \mathrm{~mm}$, respectively.

In Figure 1(b), the optical system remains physically unchanged, but the focal length of the L-Lens is changed to $f_{1}{ }^{\prime}=-222 \mathrm{~mm}$. As a result, the locations of Images $1^{\prime}, 2^{\prime}$ and $3^{\prime}$ all changed, so do the magnification ratios. Image $3^{\prime}$ is now $482.6 \mathrm{~mm}$ from the F-Lens, making it $436 \mathrm{~mm}$ closer to the driver and 1.4 times larger in size when compared to Image 3 in Figure 1(a). This produces the desired effect that a warning sign becomes larger as it gets closer to the driver. When changing the lens power of 
the L-Lens continuously between two extreme values as described the resultant image moves between the two positions in Figures 1(a) and 1(b), respectively.

\section{Results}

\subsection{Dynamic viewing effect}

A display apparatus has been set up on an optical breadboard $\left(15 \times 60 \mathrm{~mm}^{2}\right)$ according to the developed optical design as shown in Figure 1. The object used here is a speed-limit sign that is projected on a diffuser from a portable projector. The resultant image is reflected by a mirror towards a car windscreen supplied by Jaguar Land Rover, which is further reflected towards the driver. A camera (Nikon D7000) is placed at the location of the driver's eye. Two hand-written labels (' $1.6 \mathrm{~m}$ ' and ' $2 \mathrm{~m}$ ') are positioned behind the windscreen, noting the corresponding distances from the driver. The L-Lens is controlled by a Raspberry Pi 3 computer, through a USB electrical lens controller and tuned through its maximum range between -4.5 and 5.5 dioptres. The image depth of displayed content from the driver and the magnification ratio are calculated and plotted for different L-Lens powers as shown in Figure 2.

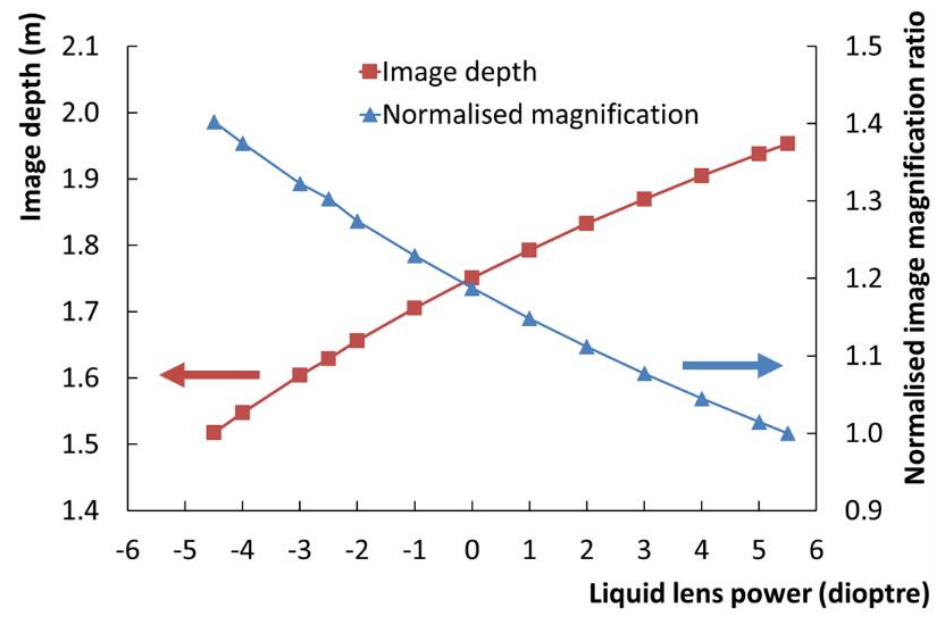

(a)

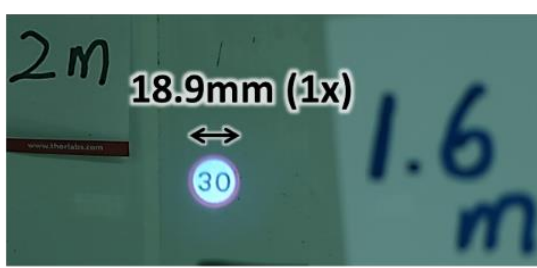

(b)

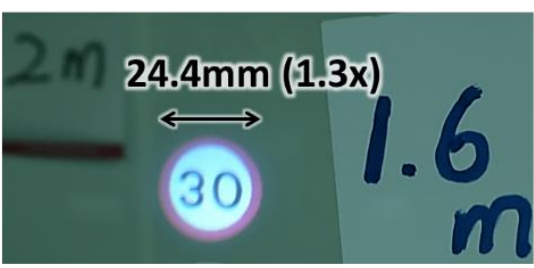

(c)

Figure 2. (a) Image depth and the normalised magnification ratio of the designed optical unit with respect to the tuning range of the L-Lens power. Images captured on the windscreen with the lens power of (b) 5.5 dioptre and (c) -2.5 dioptre, confirming the calculated image depth and size.

Two images are captured off the windscreen when the L-Lens power is 5.5 and -2.5 dioptres and shown in Figure 2(b) and 2(c) respectively. The speed-limit sign at 5.5 dioptres is in focus at $2 \mathrm{~m}$ away, indicating the large image depth. Its diameter is measured as $18.9 \mathrm{~mm}$, which is normalised to one. Likewise, when the lens power is changed to -2.5 dioptres, the speed-limit sign is in focus at $1.6 \mathrm{~m}$ away, indicating the reduced image depth. The diameter increases to $24.4 \mathrm{~mm}$, which is $\sim 1.3 \mathrm{x}$ of the 
minimum diameter that was observed at 5.5 dioptres. The maximum normalised magnification is $1.4 \mathrm{x}$ when the image is at $\sim 1.5 \mathrm{~m}$ away from the driver.

\subsection{HUD integration}

The verified optical unit is integrated into a HUD system for demonstration, the system is placed underneath the windscreen, shown as in inset image in Figure 3(a) with its top-down schematic view shown in Figure 3(b). A 2D HUD image (lane marking, round-about sign, etc.) is displayed behind the windscreen at $2 \mathrm{~m}$ away, as illustrated in Figure 3(a). The speed-limit sign is displayed at the same time with its image depth varying between $1.95 \mathrm{~m}$ to $1.52 \mathrm{~m}$. Such a dynamic visual effect can be used to attract the driver's attention in situations like speeding.

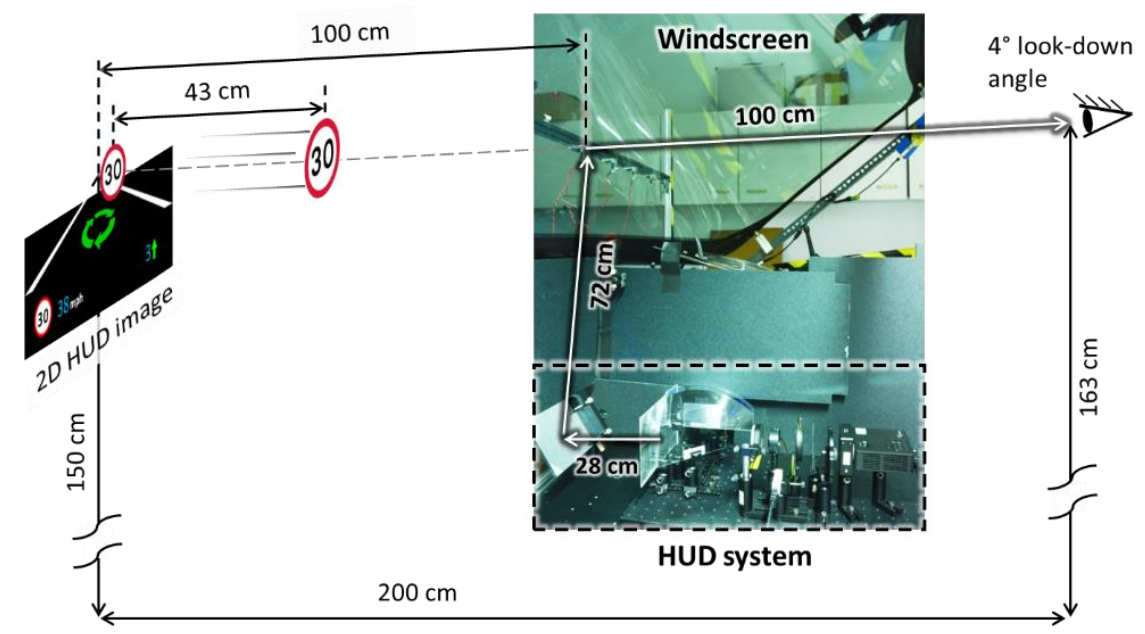

(a)

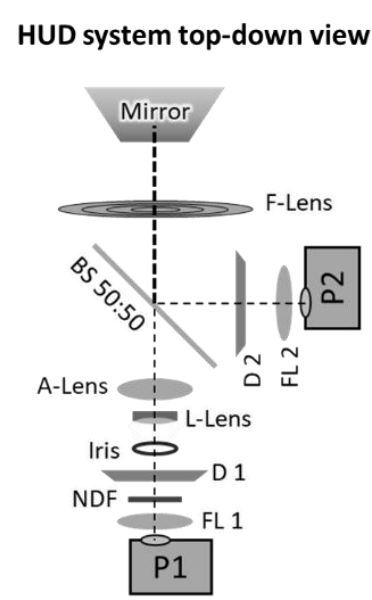

(b)

Figure 3. (a) Schematics of the HUD light path and virtual image illustrations, with an inset image showing the actual setup of the HUD system and windscreen. (b) Schematics of HUD system (top-down view) with the integrated depth-variable effect, where P1/2 is the projector one/two, FL1/2 the focussing lens one/two, NDF a neutral density filter, D1/2 the diffuser one/two, and BS a beam splitter.

In Figure 3(b), projector one (P1) generates the image of speed-limit sign on a first diffuser (D1) and projector two (P2) generates the 2D HUD image on a second diffuser (D2). Focussing lenses (FL1 \& FL2), both with a focal length of $150 \mathrm{~mm}$, are used to reduce the projection distance of P1 and P2, respectively. They also reduce the projected image size to a preferred value. A neutral density filter (NDF) is used to modulate the brightness of the depth-variable image, matching it to the 2D image. An iris is used after D1 to limit the environmental light entering the system. The L-Lens, A-Lens and F-Lens are arranged according to Figure 1. A 50:50 beam splitter (BS) combines the depth-variable images and the 2D HUD image, which both are reflected by a mirror to the windscreen.

Figure 4 shows the HUD images reflected off the windscreen with the speed-limit sign in focus at variable depths, and the superimposed 2D HUD image at $2 \mathrm{~m}$ away. From Figures $4(\mathrm{a})$ to $4(\mathrm{f})$, the 
L-Lens optical power reduces from $5.5,3.5,1.5,-0.5,-2.5$ to -4.5 dioptres. In Figure $4(a)$, both the $2 \mathrm{D}$ HUD image and the speed-limit sign are in focus, indicating the similar image depth $(\sim 2 m)$. The subsequent reduction of the L-Lens power moves the dynamic speed-limit sign closer from 1.95 to $1.52 \mathrm{~m}$ and at the same time increases its size from 22.6 to $31.2 \mathrm{~mm}$ in diameter.

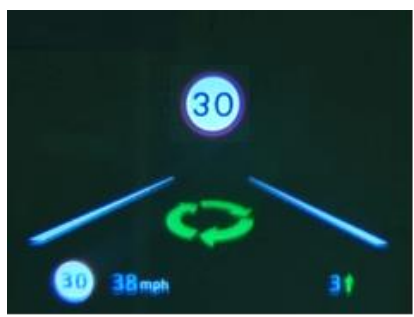

(a)

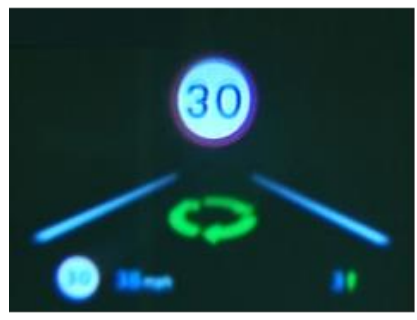

(d)

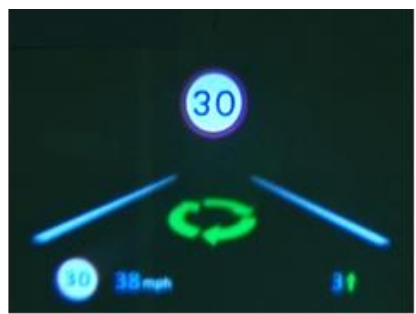

(b)

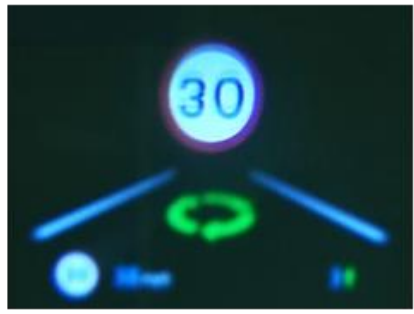

(e)

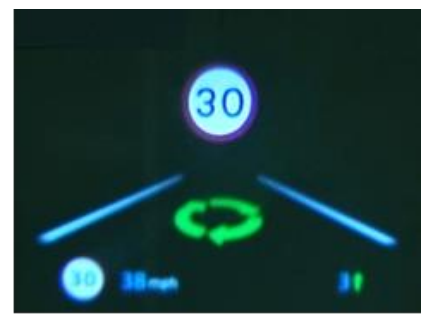

(c)

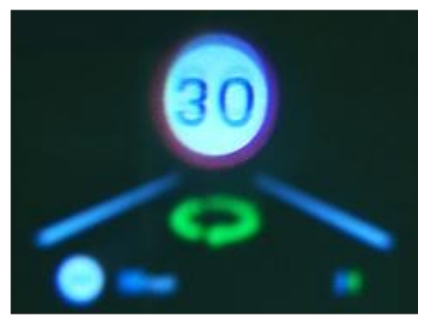

(f)

Figure 4. Images captured from the integrated HUD with the camera focused on the speed-limit sign as it moves from 1.95 to $1.52 \mathrm{~m}$ in distance, as the L-Lens power is set to (a) 5.5, (b) 3.5, (c) 1.5, (d) -0.5 , (e) -2.5 and (f) -4.5 dioptres, respectively.

The change in image size mimics a real road signage approaching the driver from 50 to 30 metres away. The image depth variation of the displayed sign differs from the actual road signage, but it leads to a larger change in eye-lens curvature of the driver. Assuming the human eye-lens can accommodate an image from $100 \mathrm{~mm}$ away to infinity, and the distance between the eye-lens and the retina is $17 \mathrm{~mm}$. As a result, the eye-lens focal length is $17 \mathrm{~mm}$ when fully relaxed and is $14.53 \mathrm{~mm}$ when viewing at $100 \mathrm{~mm}$ away. As the image moves from 1.95 to $1.52 \mathrm{~m}$, the eye-lens focal length changed by $1.66 \%$, compared with $0.16 \%$ when the signage moves from 50 to $30 \mathrm{~m}$. The displayed depth-variable image causes a larger ciliary muscle contraction, and this tends to increase driver's situation awareness. The aim of the dynamic depth-variable feature is not to display information continuously, as this can lead to distraction, but it is turned on when an immediate attention is needed from the driver.

\section{Discussion}

Displaying the dynamic sign is an effective way to attract the driver's attention, which can be useful in different applications such as for speed compliance [2]. Because of the limited L-Lens aperture $(16 \mathrm{~mm})$ in use, the area (eye-box) in which the dynamic image can be seen is restricted. 
To measure the eye-box size of the integrated HUD at the viewing plane, the camera was mounted onto a linear stage which was then fixed to a tripod, as shown in Figure 5(a). This set up allows the camera to move in both vertical and horizontal directions, so it can determine boundaries of the eyebox shown as small red circles in Figure $5(b-d)$. The measurement was carried out as the L-Lens moved between the A-Lens and the object (speed-limit sign) with other optical components fixed. The dynamic image size was set to about half-way between the minimum and maximum magnification. The virtual aperture of the L-Lens gets larger as it gets closer to the effective focal point of the combined A-Lens and F-Lens. Consequently, as the distance $d_{1}$ between the L-Lens and A-Lens changes from $28.4 \mathrm{~mm}$ (as shown in Figure 1), to $38 \mathrm{~mm}$ and to $46 \mathrm{~mm}$, the eye-box increases from $85 \mathrm{~mm}$, to $113 \mathrm{~mm}$, and to $139 \mathrm{~mm}$ in diameter as shown in Figure $5(\mathrm{~b})$ to $5(\mathrm{~d})$, respectively. Here the eye-box is circular in shape because of the circular L-Lens aperture.

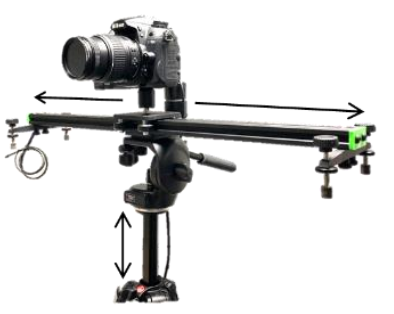

(a)

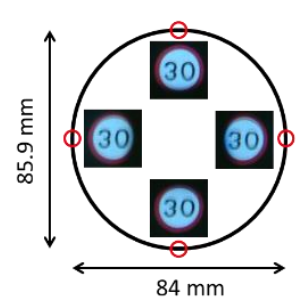

(b)

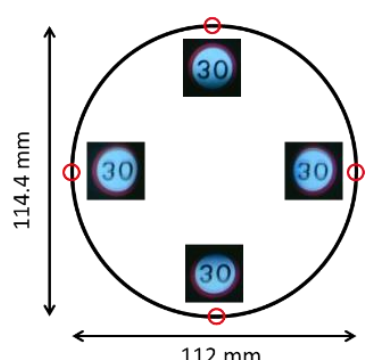

(c)

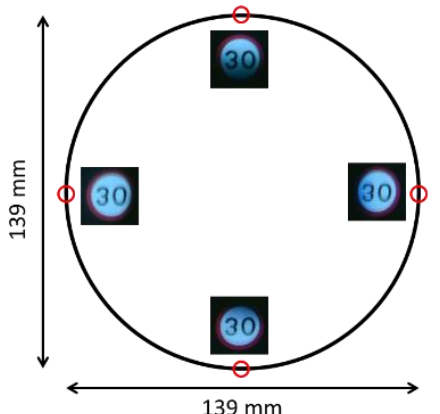

(d)

Figure 5. (a) Camera setup for the eye-box measurement. Dimensions of the circular eye boxes when the L-Lens is at (b) $28.4 \mathrm{~mm}$, (c) $38 \mathrm{~mm}$ and (d) $46 \mathrm{~mm}$ away from the A-Lens, respectively, with the inset images showing the displayed speed-limit sign at the boundaries of the corresponding eye-box.

The driver will have more room for head movement before one of the eyes loses sight of the image with a larger eye-box. However, the enlarged eye-box comes reduced image depth and magnification ranges. As shown in Figure 6 (circled data points), the image depth variation range decreases from $436 \mathrm{~mm}$ to $334 \mathrm{~mm}$ and then to $262 \mathrm{~mm}$ while the magnification range reduces from 1.4 times to 1.2 times and then to 1.1 times, as the distance $d_{1}$ were set to the three values listed in Figures $5 b, 5 c$ and $5 d$, respectively. Nevertheless, the dynamic pop-up effect remains visually very effective even at a smaller tuning range, this is to be quantified in user trials. 


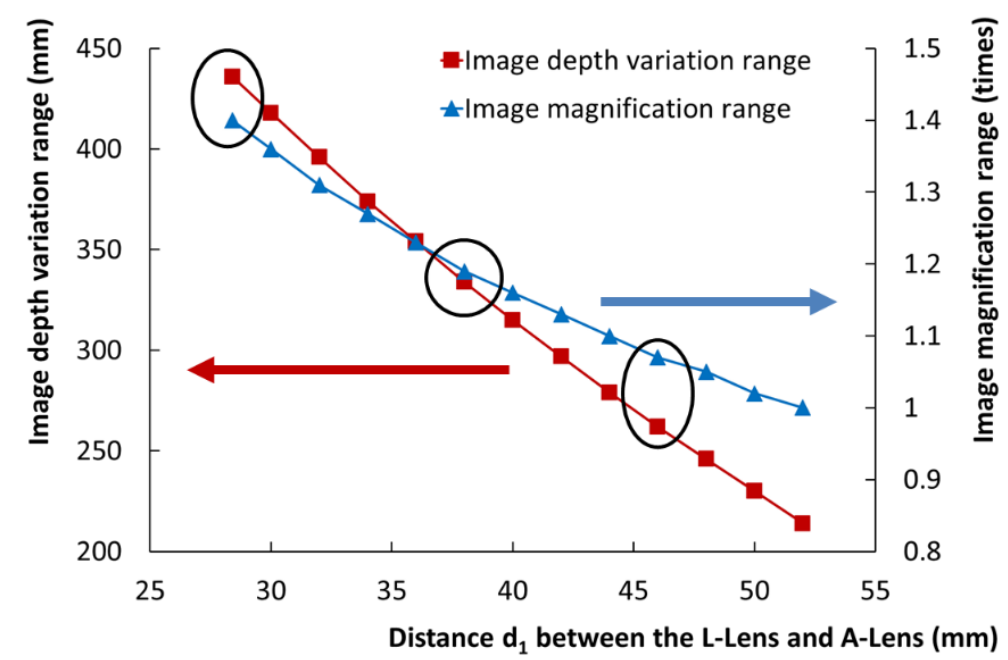

Figure 6. Image depth variation and magnification ranges with respect to the distance $d_{1}$ between the L-Lens and A-Lens.

Furthermore, it is important for HUD applications to have images displayed with a uniform brightness across the entire eye-box. This is not always the case as most of off-the-shelf projectors are off-axis projectors, hence the projected light rays have different incident angles on the diffuser which need careful consideration. In addition, the diffusers used in a HUD unit often have a limited scattering angle, so the scattered light rays end up in the eye-box for an enhanced brightness. As a result, the displayed image may appear to be brighter when viewed at some angles. This issue can be resolved by using an on-axis projector picture generation unit, or a small image on the diffuser to reduce the range of incident angles, or a flat-panel display which tends to have more uniform brightness and diverging angles. Separately, the focussing lens in front of the projector, as shown in Figure 3(b), can also converge and reduce incident angles of the light rays.

\section{Conclusions and future work}

The HUD with local dynamic and depth-variable features has been designed, developed and demonstrated, utilising an off-the-shelf electrically focus-tuneable liquid lens, with the car windscreen. The variation ranges for image depth and magnification are $436 \mathrm{~mm}$ and 1.4 times, respectively. The displayed image can be used to mimic a road-warning sign approaching the driver from 50 to 30 metres away. This approach can be used to reduce the driver's response time in detecting potential hazards so accidents can be avoided.

The eye-box of the developed HUD is limited due to the small liquid lens aperture, but it can be enlarged to accommodate the head movement during driving. As a result, the displayed image depth variation and magnification ranges are reduced, but the dynamic effect remains strong. The 
demonstrated image brightness across the entire eye-box is uniform and this is very important in applying the technology in real case scenario.

Future work is needed to reduce the dimension of the developed HUD. We aim to fold the optical path, construct the HUD with lighter, customised opto-mechanical parts, and use high brightness selfemissive micro-display panels such as microLEDs. The purpose is to fit the HUD into a prototype car so that user trials can be carried out to quantify the response time for the static and dynamic HUD warning signs. Moreover, each user might prefer slightly different settings (depth variation, magnification range, variation speed etc.), so the dynamic warning sign can be customised for each individual's preference.

\section{Acknowledgements}

This work was supported by CAPE JLR iHUD Project and UK Engineering and Physical Sciences Research Council (EPSRC) through the EPSRC Centre for Doctoral Training in Integrated Photonic and Electronic Systems (EP/L015455/1).

\section{References}

[1] WHO, Road traffic injuries, (2018). http://www.who.int/en/news-room/factsheets/detail/road-traffic-injuries (accessed April 26, 2018).

[2] S. V. Ukkusuri, K. Gkritza, X. Qian, A.M. Sadri, Best Practices for Maximizing Driver Attention to Work Zone Warning Signs, West Lafayette, 2016. doi:10.5703/1288284316338.

[3] G.-D. Tuzar, A. Van Laack, Augmented reality head-up displays : HMI impacts of different fieldof-views on user 2016. https://www.visteon.com/products/documents/augmented_reality_nov_2016_FNL.pdf.

[4] M.R. Endsley, Toward a Theory of Situation Awareness in Dynamic Systems, Hum. Factors J. Hum. Factors Ergon. Soc. 37 (1995) 32-64. doi:10.1518/001872095779049543.

[5] H.S. Park, M.W. Park, K.H. Won, K.H. Kim, S.K. Jung, In-Vehicle AR-HUD system to provide driving-Safety information, ETRI J. 35 (2013) 1038-1047. doi:10.4218/etrij.13.2013.0041.

[6] A. Pauzie, Head Up Display in Automotive: A New Reality for the Driver, in: Springer Int. Publ. Switz. 2015, 2015: pp. 505-516. doi:10.1007/978-3-319-20889-3.

[7] K. Bengler, K. Dietmayer, B. Farber, M. Maurer, C. Stiller, H. Winner, Three decades of driver assistance systems: Review and future perspectives, IEEE Intell. Transp. Syst. Mag. 6 (2014) 622. doi:10.1109/MITS.2014.2336271.

[8] J.A. Betancur, J. Villa-Espinal, G. Osorio-Gómez, S. Cuéllar, D. Suárez, Research topics and implementation trends on automotive head-up display systems, Int. J. Interact. Des. Manuf. (2016) 1-16. doi:10.1007/s12008-016-0350-3. 
[9] Z. Medenica, A.L. Kun, T. Paek, O. Palinko, Augmented reality vs. street views, Proc. 13th Int. Conf. Hum. Comput. Interact. with Mob. Devices Serv. - MobileHCl '11. (2011) 265. doi:10.1145/2037373.2037414.

[10] M. WHEELER, HUD Systems: Augmented Reality Is Coming to Your Windshield, Photonics Spectra.

(2016). https://www.photonics.com/a58298/HUD_Systems_Augmented_Reality_Is_Coming_to_Your (accessed April 26, 2018).

[11] MarketsAndMarkets, Head-Up Display Market by Component (Video Generator, Projector/Projection Unit, Display Unit, Software), Type (Conventional HUD, AR-Based HUD), Application (Aviation, Automotive), and Geography - Global Forecast to 2023, (2018). https://www.marketsandmarkets.com/Market-Reports/head-up-display-hud-market684.html?gclid=CjOKCQiA5t7UBRDaARIsAOreQtjEE5Sphkor82XvK4sUftK6mSGIXxvTvYzxOBzts xKdLY7M588wdVgaAjyVEALw_wcB (accessed April 26, 2018).

[12] GrandViewResearch, Head Up Display Market Worth \$13.5 Billion By 2025 | CAGR: 25.9\%, (2017). https://www.grandviewresearch.com/press-release/global-head-up-display-hudmarket (accessed April 26, 2018).

[13] J.L. Gabbard, G.M. Fitch, H. Kim, Behind the glass: Driver challenges and opportunities for AR automotive applications, Proc. IEEE. 102 (2014) 124-136. doi:10.1109/JPROC.2013.2294642.

[14] M.L. Rusch, M.C. Schall, P. Gavin, J.D. Lee, J.D. Dawson, S. Vecera, M. Rizzo, Directing driver attention with augmented reality cues, Transp. Res. Part F Traffic Psychol. Behav. 16 (2013) 127-137. doi:10.1016/j.trf.2012.08.007.

[15] WayRay, WayRay raises $\$ 80 \mathrm{M}$ in a Round $\mathrm{C}$ led by Porsche and joined by Hyundai Motor, JVCKENWOOD, and other investors, aiming to become a \$ 1B company next year., (2018). https://wayray.com/pressarea/pressrelease/2018\#roundC.

[16] Continental, Press Release Revolutionizing Head-up Displays - Continental Increases Investment in DigiLens, (2018) 1-2. https://www.digilens.com/press-release/revolutionizinghead-displays-continental-increases-investment-digilens/.

[17] J. Wickens, Christopher D. Long, Object versus space-based models of visual attention: Implications for the design of head-up displays., J. Exp. Psychol. Appl. 1 (1995) 179-193. doi:http://dx.doi.org/10.1037/1076-898X.1.3.179.

[18] J. Caird, W. Horrey, C. Edwards, Effects of conformal and non-conformal vision enhancement systems on older driver performance, Transp. Res. Rec. J. Transp. Res. Board. 1759 (2001) 3845. doi:https://doi.org/10.3141/1759-05.

[19] D.R. Tufano, Automotive HUDs: The Overlooked Safety Issues, Hum. Factors J. Hum. Factors Ergon. Soc. 39 (1997) 303-311. doi:10.1518/001872097778543840.

[20] Z. McDonald, Augmented Reality HUDs: Warning Signs and Drivers' Situation Awareness, Rochester Institute of Technology, 2016.

[21] R. Azuma, R. Behringer, S. Feiner, S. Julier, B. Macintyre, Recent Advances in Augmented Reality, IEEE Comput. Graph. Appl. 2011 (2001) 1-27. doi:10.4061/2011/908468.

[22] D.L. Fisher, K.C. Tan, Visual displays: The highlighting paradox, Hum. Factors. 31 (1989) 17-30. doi:10.1177/001872088903100102.

[23] A. Doshi, S.Y. Cheng, M.M. Trivedi, A novel active heads-up display for driver assistance, IEEE Trans. Syst. Man, Cybern. Part B Cybern. 39 (2009) 85-93. doi:10.1109/TSMCB.2008.923527. 
[24] H. Yoo, O. Tsimhoni, H. Watanabe, P. Green, R. Shah, Display of HUD Warnings to Drivers: Determining an Optimal Location, Yokosuka, Japan, 1999.

[25] Continental, Continental head up display, (2014). http://continental-head-up-display.com/.

[26] S. Liu, H. Hua, Time-multiplexed dual-focal plane head-mounted display with a liquid lens, Opt. Lett. 34 (2009) 1642-1644. doi:10.1364/OL.34.001642.

[27] P. V. Johnson, J.A. Parnell, J. Kim, C.D. Saunter, G.D. Love, M.S. Banks, Dynamic lens and monovision 3D displays to improve viewer comfort, Opt. Express. 24 (2016) 11808. doi:10.1364/OE.24.011808.

[28] G.D. Love, D.M. Hoffman, P.J.W. Hands, J. Gao, A.K. Kirby, M.S. Banks, High-speed switchable lens enables the development of a volumetric stereoscopic display, Opt. Express. 17 (2009) 15716. doi:10.1364/OE.17.015716.

[29] Optotune, Electrically tunable large aperture lens Datasheet: EL-16-40-TC, (2018) 1-9. 